\title{
The roles of chronic pressure and volume overload states in induction of arrhythmias: An animal model of physiologic sequelae after repair of tetralogy of Fallot
}


Robert Waibel, ${ }^{\text {b }}$ Richard F. Ittenbach, PhD, ${ }^{c}$ Ronn E. Tanel, MD, ${ }^{d}$ Victoria L. Vetter, MD, ${ }^{d}$ and Larry A. Rhodes, MD

From the Division of Cardiology at Children's Medical Center at Dallas and the University of Texas Southwestern Medical


Cardiothoracic Surgery, ${ }^{\mathrm{b}}$ Biostatistic and Data Management Core, ${ }^{\mathrm{c}}$ and Cardiology ${ }^{\mathrm{d}}$ at The Children's Hospital of Philadelphia and the University of Pennsylvania School of Medicine, Philadelphia, $\mathrm{Pa}$

This study was supported in part by a postdoctoral research fellowship award (I.Z.) from the American Heart Association (award ID no. 0325738U). Additional support for this project was provided through the Daniel M. Tabas Endowed Chair in Pediatric Cardiothoracic Surgery (J.W.G.).

Received for publication March 4, 2005; revisions received July 20, 2005; accepted for publication Aug 16, 2005.

Address for reprints: Ilana Zeltser, MD, Children's Medical Center at Dallas, The University of Texas Southwestern, 1935 Motor St, Dallas, TX 75235 (E-mail: Ilana. zeltser@childrens.com).

J Thorac Cardiovasc Surg 2005;130:1542-8

$0022-5223 / \$ 30.00$

Copyright (C) 2005 by The American Association for Thoracic Surgery

doi:10.1016/j.jtcvs.2005.08.034
Objective: Sudden death occurs in as many as $8 \%$ of patients after repair of tetralogy of Fallot and has been attributed to arrhythmias. The purpose of this study was to establish an animal model to evaluate the individual contribution of different physiologic sequelae after tetralogy of Fallot repair in the development of late-onset arrhythmias.

Methods: Forty-nine piglets were divided into 5 groups: (1) pulmonary artery band; (2) pulmonary valvotomy; (3) pulmonary artery band plus pulmonary valvotomy; (4) infundibular scar; and (5) age-matched control animals. Baseline and follow-up electrocardiograms were obtained and recorded, as well as changes in QRS duration. A total of 45 animals underwent hemodynamic evaluation and programmed electrical stimulation at 5.6 months postoperatively.

Results: Sustained ventricular tachyarrhythmias (ventricular tachycardia/ventricular fibrillation) were induced in $31.1 \%$, and atrial arrhythmias were induced in $33.3 \%$. The pulmonary valvotomy group was 30 times more likely to evidence arrhythmias than control animals for sustained ventricular tachycardia/ventricular fibrillation, as well as atrial arrhythmias $(P=.01)$. The pulmonary artery band group was 15 times more likely to evidence atrial arrhythmias than control animals $(P=.02)$. Prolonged QRS duration was predictive of inducibility of both atrial arrhythmias $(P<.01)$ and sustained ventricular tachycardia/ventricular fibrillation $(P=.01)$. Mean right atrial $(P=.01)$ and capillary wedge $(P=.01)$ pressures predicted atrial arrhythmia inducibility. Right ventricular end-diastolic pressure predicted atrial arrhythmia $(P=.01)$ and sustained ventricular tachycardia/ventricular fibrillation inducibility $(P=.05)$. Right ventricular systolic pressure did not predict inducibility of either atrial arrhythmias $(P=.10)$ or sustained ventricular tachycardia/ventricular fibrillation $(P=.94)$.

Conclusions: Chronic right ventricular volume overload resulted in an increased incidence of inducible ventricular and atrial arrhythmias.

$\mathrm{T}$ etralogy of Fallot (TOF) is the most common cause of cyanotic congenital heart disease, with a prevalence rate of 0.26 to 0.48 cases per 1000 live births. ${ }^{1}$ In the current surgical era, complete intracardiac repair for TOF is commonly performed during infancy, with actuarial survival rates of $95 \%$ after 5 years and $93 \%$ after 30 years. ${ }^{2}$ Although the long-term mortality rate after TOF repair is low, late sudden death occurs in approximately $2 \%$ to $8 \%$ of all postoperative patients and has been attributed to ventricular arrhythmias. ${ }^{3-12}$ The desire to identify at-risk patients has prompted investigation into potential risk factors leading to the development of sustained ventricular tachycardia/ventricular fibrillation (VT/ VF). Conflicting data exist regarding the relationship between sustained VT/VF and right ventricular (RV) systolic hypertension, diastolic hypertension, or both ${ }^{12-17}$ and 


$$
\begin{aligned}
& \text { Abbreviations and Acronyms } \\
& \begin{aligned}
\mathrm{AA} & =\text { atrial arrhythmia } \\
\mathrm{ECG} & =\text { electrocardiogram } \\
\mathrm{OR} & =\text { odds ratio } \\
\mathrm{PAB} & =\text { pulmonary artery band } \\
\mathrm{PR} & =\text { pulmonary regurgitation } \\
\mathrm{PV} & =\text { pulmonary valvotomy } \\
\mathrm{RV} & =\text { right ventricular } \\
\mathrm{SCD} & =\text { sudden cardiac death } \\
\mathrm{TOF} & =\text { tetralogy of Fallot } \\
\mathrm{VF} & =\text { ventricular fibrillation } \\
\mathrm{VT} & =\text { ventricular tachycardia }
\end{aligned}
\end{aligned}
$$

RV outflow tract scar or aneurysm. ${ }^{18-21}$ More recently, attention has been focused on the contribution of increased RV volume caused by pulmonary regurgitation (PR) and its effect on prolongation of ventricular depolarization. 9,17,22-24

Studies designed to identify predictive risk factors for sudden cardiac death (SCD) in patients with TOF have been retrospective and have involved cohorts of patients with a variety of hemodynamic lesions. As such, it has been difficult to analyze individual risk factors and their potential roles in the development of lethal arrhythmias. A viable and reproducible animal model was created to mimic physiologic sequelae similar to those found after surgical repair of TOF to evaluate these risk factors independently. During cardiac catheterization and programmed electrical stimulation, the electrophysiologic effects of RV hypertension, PR, and scarring caused by a surgical incision in the RV outflow tract were investigated with respect to arrhythmia inducibility.

\section{Methods}

\section{Animal Model}

This study surgically established an animal model in which 4 conditions represented the physiologic sequelae after TOF repair: RV hypertrophy caused by pulmonary stenosis, RV dilatation caused by PR, RV scar formation (similar to a ventriculotomy), and a combination of all 3 conditions. The effect of each of these factors was evaluated 5 to 6 months postoperatively. The animal model consisted of 4 surgical groups and one control group: (1) pulmonary artery band (PAB) placement plus infundibular scar; (2) pulmonary valvotomy (PV) plus infundibular scar; (3) PAB placement plus PV plus infundibular scar (PAB/PV); (4) infundibular scar only (Scar group), with preservation of the integrity of the valve annulus; and (5) a control group consisting of age-matched animals without any intervention purchased from a commercial vendor at 6 months of age.

\section{Anesthesia-sedation}

Thirty-five 3- to 7-day-old piglets $(2-5 \mathrm{~kg})$ were obtained from a commercial vendor and studied according to a protocol approved by the Institutional Animal Care and Use Committee of the Joseph
Stokes Research Institute. The protocol is in compliance with the National Academy of Science's "Guide for the Care and Use of Laboratory Animals" (National Institutes of Health publication no. 96-03, revised 1996). In a fasting state, newborn piglets were administered a premedication consisting of ketamine, $30 \mathrm{mg} / \mathrm{kg}$, and acepromazine, $1.2 \mathrm{mg} / \mathrm{kg}$, administered intramuscularly. The animals were mask ventilated with isoflurane, $2 \%$ to $5 \%$, before endotracheal intubation. Anesthesia was maintained throughout the procedure with $1 \%$ to $2 \%$ isoflurane. An intravenous catheter was placed in an ear vein, and cefazolin, $20 \mathrm{mg} / \mathrm{kg}$, was administered. Peripheral oxygen saturation, heart rate, and blood pressure were monitored noninvasively throughout the procedure. After the procedure, the animals were extubated and received supplemental oxygen in an incubator until room air was tolerated. Postoperative pain management included buprenorphine (Buprenex), $0.1 \mathrm{mg} / \mathrm{kg}$ twice a day for 2 days. Animals were bottle or tube fed until they could self-feed.

\section{Intraoperative Procedure}

All surgical study animals received a muscle-sparing left thoracotomy between the fifth or sixth interspace and were then divided into one of the 4 aforementioned surgical groups. In the PAB and $\mathrm{PAB} / \mathrm{PV}$ groups, the $\mathrm{PAB}$ was placed by means of circumferential dissection of the pulmonary artery $1 \mathrm{~cm}$ distal to the valve annulus. After dissection, a $1 / 4-\mathrm{cm}$ umbilical tape was passed around the artery and secured for a final diameter of approximately $1 \mathrm{~cm}$. In the $\mathrm{PV}$ and $\mathrm{PAB} / \mathrm{PV}$ groups, a PV was performed by means of placement of a side-biting Satinsky vascular clamp longitudinally across the pulmonary valve annulus without obstruction of the RV outflow tract. A 2-cm incision was made longitudinally across the pulmonary annulus, and 2 pulmonary valve leaflets were excised. Next, a 2-cm-long elliptically shaped polytetrafluorethylene patch was sewn in place with 6-0 nonabsorbable monofilament sutures across the annulus to ensure complete disruption of valve integrity. All surgical study groups, including the Scar group, also received an infundibular scar made by placement of a side-biting vascular clamp placed longitudinally over the infundibulum without interruption of the pulmonary valve apparatus. A 1-cm full-thickness incision was made longitudinally over the infundibulum up to but not including the valve annulus and then repaired with the placement of a 1-cm-long elliptically shaped polytetrafluorethylene patch to ensure scar formation. At the completion of each procedure, hemostasis was obtained, and the thoracotomy was closed over a fully expanded left lung with 2-0 absorbable sutures.

\section{Noninvasive Evaluation}

On postoperative days 90 and 180, the animals were sedated for a transthoracic echocardiogram. Two-dimensional imaging and color Doppler flow analysis were used to determine the presence of pulmonary stenosis or pulmonary insufficiency. A 12lead electrocardiogram (ECG) was performed preoperatively and again before cardiac catheterization, except in the control animals, in which ECGs were obtained only before cardiac catheterization, to determine the duration of the QRS complex in leads I, II, V1, and V2. The maximum QRS duration was used for statistical analysis. 
TABLE 1. Descriptive statistics and surgical group comparisons for selected hemodynamic variables

\begin{tabular}{|c|c|c|c|c|c|c|c|c|c|c|c|}
\hline \multirow[b]{2}{*}{$\begin{array}{l}\text { Hemodynamic } \\
\text { outcome }\end{array}$} & \multicolumn{2}{|c|}{ PAB group } & \multicolumn{2}{|c|}{ PV group } & \multicolumn{2}{|c|}{ PAB/PV group } & \multicolumn{2}{|c|}{ Scar group } & \multicolumn{2}{|c|}{ Control group } & \multirow[b]{2}{*}{$\begin{array}{c}P \\
\text { value }\end{array}$} \\
\hline & $\begin{array}{l}\text { Mean } \\
\text { (SD) }\end{array}$ & $\begin{array}{r}\text { Median } \\
\text { (range) }\end{array}$ & $\begin{array}{l}\text { Mean } \\
\text { (SD) }\end{array}$ & $\begin{array}{l}\text { Median } \\
\text { (range) }\end{array}$ & $\begin{array}{l}\text { Mean } \\
\text { (SD) }\end{array}$ & $\begin{array}{l}\text { Median } \\
\text { (range) }\end{array}$ & $\begin{array}{l}\text { Mean } \\
\text { (SD) }\end{array}$ & $\begin{array}{l}\text { Median } \\
\text { (range) }\end{array}$ & $\begin{array}{l}\text { Mean } \\
\text { (SD) }\end{array}$ & $\begin{array}{l}\text { Median } \\
\text { (range) }\end{array}$ & \\
\hline QRS & $84.3(5.3)$ & $80.0(80-90)$ & $83.3(5.2)$ & $80.0(80-90)$ & $74.0(15.2)$ & $70.0(60-90)$ & $60.8(8.6)$ & $60.0(50-70)$ & $71.4(6.6)$ & $70.0(60-80)$ & $<.01$ \\
\hline SBP & $74.0(4.0)$ & $75.0(65-77)$ & $75.8(11.0)$ & $75.0(63-90)$ & $80.0(7.1)$ & $80.0(70-90)$ & 77.1 (10.2) & $75.0(56-92)$ & $80.4(9.3)$ & $79.0(64-98)$ & .48 \\
\hline RAP & $9.6(4.7)$ & $9.0(3-16)$ & $7.7(3.6)$ & $8.0(3-12)$ & $8.6(1.5)$ & $9.0(6-10)$ & $4.5(3.3)$ & $4.0(1-12)$ & $2.4(1.5)$ & $2.0(1-5)$ & $<.01$ \\
\hline RVSP & $57.7(12.1)$ & $60.0(43-72)$ & $31.7(13.6)$ & $24.5(20-54)$ & $57.0(18.6)$ & $50.0(45-90)$ & $22.1(8.1)$ & $24.0(8-37)$ & $18.0(4.5)$ & $17.5(12-26)$ & $<.01$ \\
\hline RVEDP & $10.9(4.8)$ & $12.0(3-16)$ & $12.2(4.4)$ & $13.0(6-17)$ & $11.4(1.5)$ & $11.0(10-13)$ & $5.7(3.9)$ & $5.0(2-12)$ & $3.9(1.0)$ & $4.0(2-5)$ & $<.01$ \\
\hline PCWP & $14.4(5.1)$ & $15.0(6-23)$ & $9.7(3.2)$ & $10.0(6-14)$ & $10.6(3.4)$ & $11.0(5-14)$ & $7.5(5.7)$ & $6.0(1-18)$ & $6.0(2.1)$ & $6.0(3-10)$ & $<.01$ \\
\hline
\end{tabular}

Note: $\mathrm{n}=45$. QRS duration is measured in milliseconds, and pressures are recorded in millimeters of mercury. $P A B$, Pulmonary artery band; $P V$, pulmonary valvotomy; $P A B / P V$, pulmonary artery band plus pulmonary valvotomy; $S c a$, infundibular scar; $S D$, standard deviation; $S B P$, systolic blood pressure; $R A P$, right atrial mean pressure; RVSP, right ventricular systolic pressure; RVEDP, right ventricular end-diastolic pressure; $P C W P$, pulmonary capillary wedge pressure.

\section{Invasive Evaluation}

At a mean interval of 5.6 months after surgical intervention, the approximate equivalence of swine adulthood, ${ }^{25}$ the animals were transferred to the animal catheterization laboratory, where they were sedated, intubated, and given general anesthesia, as described above. Vascular access was obtained percutaneously through the femoral vein and artery and through cut-down to expose the internal jugular vein. Pressure measurements were recorded in the right atrium, right ventricle, pulmonary artery, and systemic artery.

Diagnostic electrophysiologic testing was performed according to the established guidelines outlined in previous studies. ${ }^{6,17,19,26}$ Quadrapolar electrode catheters were placed in the right atrium and right ventricle for recording and stimulation. The programmed electrical stimulation protocol included measurement of the following: (1) baseline intracardiac intervals; (2) sinus node recovery time at 2 paced cycle lengths; (3) Wenckebach cycle length; and (4) atrioventricular nodal effective refractory period. Inducibility of atrial arrhythmias (AAs) was assessed with atrial single and double premature extrastimuli introduced at 2 drive-train cycle lengths, followed by burst atrial pacing. Inducibility of ventricular arrhythmias was assessed with RV apex single and double premature ventricular extrastimuli at 2 drive-train cycle lengths, followed by burst ventricular pacing with decrementing cycle lengths to ventricular refractoriness or to a minimal cycle length of $200 \mathrm{~ms}$. The ventricular stimulation protocol was performed at the RV apex. A test result was considered positive if an atrial tachycardia, VT/VF, or both of greater than 6 beats in duration was induced.

\section{Statistical Analysis}

Data analysis occurred in 3 distinct phases. Phase I consisted of generating frequency counts and measures of central tendency, variability, and association for all variables in the data set, with special attention given to 6 specific hemodynamic variables: QRS duration, systemic blood pressure, right atrial mean pressure, RV systolic pressure, RV end-diastolic pressure, and pulmonary capillary wedge pressure. Because of the small sample sizes and the nonnormative nature of the distributions, all summary data reported here are presented as means and medians with their corresponding standard deviations and ranges (Table 1).

Phase II consisted of 2 different subsets of single covariate logistic regression models. In the first subset 2 logit models were specified and tested, specifically to test the relationship between surgical procedure and the presence or absence of arrhythmias, first for AA and then for VT/VF. Surgical procedure was dummy coded as a categoric variable with 5 levels (ie, none [reference control group], PAB group, PV group, PAB/PV group, and surgical scar in the outflow tract [Scar group]). In the second subset 2 sets of single covariate logit models were specified and tested with different electrophysiologic and hemodynamic variables to identify potential risk factors for arrhythmias. Higher-order interactions between statistically significant risk factors obtained in the single covariate equations and surgical procedure were investigated but not pursued because of the small sample sizes.

Phase III consisted primarily of secondary analyses in which the 6 aforementioned hemodynamic measurements were compared across the 5 surgical groups. Group differences were compared with respect to 6 specific outcomes (ie, QRS duration, systemic blood pressure, right atrial mean pressure, RV systolic pressure, RV diastolic pressure, and pulmonary capillary wedge pressure) by using the Kruskal-Wallis technique. Follow-up tests were then conducted between animals in each of the 4 experimental groups and the control group by using the Wilcoxon rank sum test as warranted. Nonparametric tests were deemed most appropriate for these data, given the small sample sizes and the nonnormative nature of the distributions. Because of the pilot nature of the study, the hypothesis-wise error rate was not adjusted beyond an $\alpha$ level of .05. All analyses were conducted with STATA 8.0 software (STATA Corp, College Station, Tex).

\section{Results}

\section{Subject Population}

Thirty-five animals underwent an operative procedure, 31 of which survived to invasive evaluation and were compared with age-matched control animals. Postoperative deaths occurred in 2 animals in the PAB/PV group and 1 each in the $\mathrm{PV}$ and Scar groups. The mean age at the time of the invasive evaluation was 5.6 months (standard deviation, 1.3 months). Those animals that underwent catheterization before 5 months of age were identified by the veterinarian as having concerning medical issues, including poor feeding 


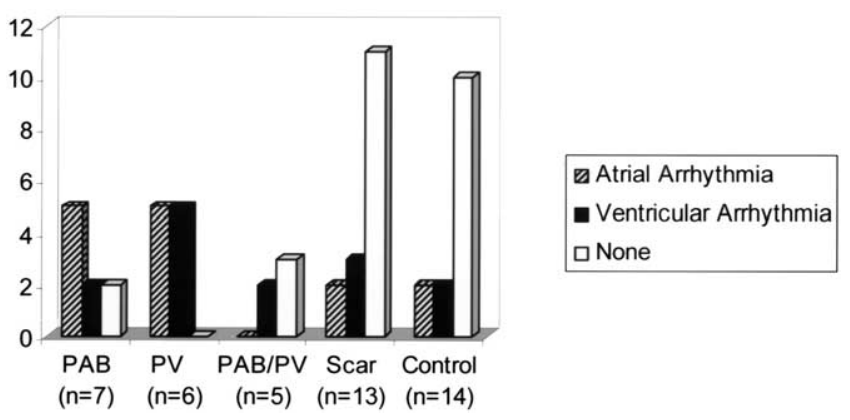

Figure 1. Arrhythmia according to surgical group. PAB, Pulmonary artery band group; $P V$, pulmonary valvotomy group; $P A B / P V$, Pulmonary artery band plus pulmonary valvotomy group; Scar, infundibular scar-only group.

and decreased activity. Seven animals had PAB and scar, 6 animals had PV and scar, 5 animals had combined PAB/PV plus scar, 13 animals had scar only, and 14 were control animals. There were 3 iatrogenic deaths, 1 animal in each of the PAB, Scar, and PAB/PV groups, during the electrophysiologic study before the ventricular stimulation protocol. Causes of iatrogenic death included asynchronous defibrillation of atrial fibrillation $(\mathrm{n}=2)$ and excessive energy used while obtaining ventricular thresholds $(n=1)$.

\section{Electrophysiologic and Hemodynamic Results}

The mean QRS duration during infancy before the procedure was $44.4 \pm 5.0 \mathrm{~ms}$. At the time of cardiac catheterization, the mean QRS duration was $72.2 \pm 11.8 \mathrm{~ms}$, with a QRS duration of $80 \mathrm{~ms}$ or greater in $19(42.2 \%)$ animals. The mean change in the QRS duration was $27.8 \pm 12.0 \mathrm{~ms}$. Catheterizations were performed on 31 surgically manipulated animals at a mean age of $5.9 \pm 1.5$ months (range, 3.2-8.1 months) and on 14 control animals at a mean age of $5.5 \pm 0.5$ months. RV systolic and end-diastolic pressures were $31.5 \pm 19.1$ and $7.4 \pm 4.6 \mathrm{~mm} \mathrm{Hg}$, respectively. All animals with $\mathrm{PAB}$ and animals with $\mathrm{PAB} / \mathrm{PV}$ demonstrated greater than one-half systemic RV pressures. Mean filling pressures in the right atrium were $5.5 \pm 4.0 \mathrm{~mm} \mathrm{Hg}$, and pulmonary capillary wedge pressures were $8.8 \pm 4.9 \mathrm{~mm}$ Hg. Data stratified according to individual surgical groups and control animals are shown in Table 1.

\section{Risk Factors for Arrhythmia Vary by Surgical Substrate}

Data on risk factors for arrhythmia by surgical substrate are shown in Figure 1. Inducible VT/VF occurred in 14 (31.1\%) of the animals undergoing operations. Thirteen of the 14 animals had inducible and hemodynamically unstable ventricular arrhythmias, 7 with polymorphic VT that degenerated to VF and 6 with VF. The 14th animal had inducible
TABLE 2. Logistic regression results for arrhythmia risk factor models

\begin{tabular}{lcccc}
\hline $\begin{array}{l}\text { Model and surgical } \\
\text { group }\end{array}$ & $\begin{array}{c}\boldsymbol{\beta} \\
\text { coefficient }\end{array}$ & $\begin{array}{c}\text { Wald } \\
\text { statistic (z) }\end{array}$ & $\begin{array}{c}\text { Odds ratio } \\
(\mathbf{9 5 \%} \mathbf{C l})\end{array}$ & $\begin{array}{c}\boldsymbol{P} \\
\text { value }\end{array}$ \\
\hline $\begin{array}{l}\text { Atrial model } \\
\text { Control* }\end{array}$ & & & & \\
PAB & 2.71 & 2.39 & $15.0(1.6-138.2)$ & .02 \\
Scar & 0.59 & 0.58 & $1.8(0.2-13.0)$ & .56 \\
PV & 3.40 & 2.55 & $30.0(2.2-411.0)$ & .01 \\
PAB/PV† & & & & \\
Intercept & -1.79 & -2.35 & & \\
Ventricular model & & & & \\
Control & & & & .44 \\
PAB & 0.87 & 0.77 & $2.4(0.36-22.1)$ & .44 \\
Scar & 0.59 & 0.58 & $1.8(0.2-13.0)$ & .56 \\
PV & 3.40 & 2.55 & $30.0(2.2-411.0)$ & .01 \\
PAB/PV & 1.39 & 1.16 & $4.0(0.4-41.2)$ & .24 \\
Intercept & -1.79 & -2.35 & & \\
\hline
\end{tabular}

Note: $\mathrm{n}=45$ animals. Outcome of interest is either atrial arrhythmia (atrial model) or ventricular tachycardia/ventricular fibrillation (ventricular model). $C l$, Confidence interval; $P A B$, pulmonary artery band; Scar, infundibular scar; $P V$, pulmonary valvotomy; $P A B / P V$, pulmonary artery band plus pulmonary valvotomy. *Reference group for logistic comparisons. $\dashv \mathrm{PAB} / \mathrm{PV}$ group was dropped from the model because of insufficient variability.

monomorphic VT lasting 2.1 seconds in duration. There was no statistical difference between the surgical intervention and the type of ventricular arrhythmia induced.

There was a relationship between the type of operation and the ability to induce VT $(P=.04)$. VT/VF was induced in $5(83 \%)$ of 6 animals in the PV group but in only 3 of 13 animals in the Scar group and 2 animals in each of the PAB $(\mathrm{n}=7), \mathrm{PAB} / \mathrm{PV}(\mathrm{n}=5)$, and control $(\mathrm{n}=14)$ groups. Using a logistic regression model with surgical treatment group as a potential risk factor for VT/VF, animals who underwent PV were more likely to have inducible VT/VF compared with the control group (odds ratio [OR], 30; $P=$ .01 ; Table 2]. Because of the small number of animals in each group, significant intergroup comparisons were not performed.

Inducible AAs occurred in 33.3\% $(n=15)$ of the animals undergoing operations but varied markedly among the different surgical groups. For example, $5(71 \%)$ of 7 animals in the PAB group and $5(83 \%)$ of 6 animals in the PV group had inducible AA, whereas only $3(23.1 \%)$ of 13 animals in the Scar group and 2 (14\%) of 14 control animals had inducible AA. No pig in the combined PAB/PV group had an inducible AA. When examined by using a singlecovariate logit equation, there was a statistically significant relationship between surgical treatment and the presence or absence of AA $(P<.01)$, with a 15 -fold increase of risk for animals in the PAB group and a 30 -fold increase for animals 


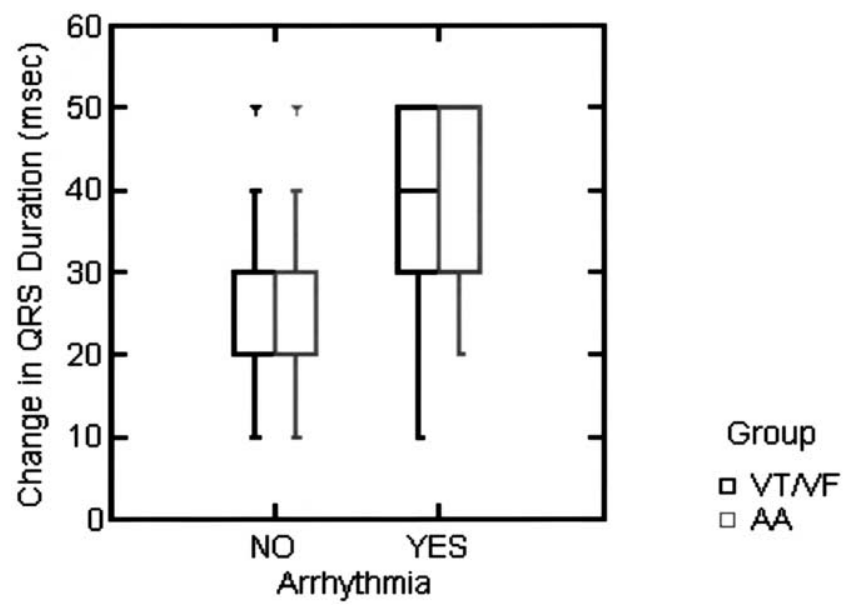

Figure 2. Relationship between change in ORS duration and arrhythmia inducibility. VT/VF, Ventricular tachycardia/ventricular fibrillation; $\boldsymbol{A} \boldsymbol{A}$, atrial arrhythmia.

in the PV group compared with that seen in the control group (Table 2).

\section{Prognostic Significance of the QRS Duration}

Logistic regression analysis demonstrated that a prolonged QRS duration was predictive of inducibility of AA $(P<$ $.01)$ and VT/VF $(P=.01)$. Of note, there was a statistically significant difference in the QRS durations for all surgical groups when compared with the control animals $(P<.01)$, except for the PAB/PV group $(P=.92)$. The control animals did not have neonatal ECGs but can be presumed to have similar QRS durations to the neonatal animals before undergoing the surgical procedures. For the entire cohort, the change in the QRS duration from before surgical intervention to the time of catheterization was $27.8 \pm 12.0 \mathrm{~ms}$. By using single covariate logistic regression models, a greater change in QRS duration predicted inducibility of AA $(P=.01)$ and VT/VF $(P=.01$, Figure 2$)$.

\section{Importance of Increased RV Pressures}

RV systolic pressure did not predict inducibility of either AA $(P=.10)$ or VT/VF $(P=.94)$. However, RV systolic pressures were significantly increased in all surgical groups but one when compared with those in the control group ( $P$ $<.01$, Table 1). The animals in the Scar group were the lone exception $(P=.12)$. With respect to $\mathrm{RV}$ end-diastolic pressure specifically, a statistically significant relationship was observed between RV end-diastolic pressure and AA ( $P$ $=.01)$, with a strong trend toward significance for VT/VF $(P=.06)$. Given this trend, when the iatrogenic causes of VT/VF are excluded from the analysis, a statistically significant relationship between an increased RV end-diastolic pressure and inducibility of VT/VF was observed $(P=.05)$.
Similar to the pattern of findings for RV systolic pressure, a statistically significant difference in RV end-diastolic pressure was observed between all of the surgical groups but one and the control group $(P<.01)$. Animals in the Scar group again proved to be the lone exception $(P=.27)$.

\section{Role of Atrial Compliance}

Right atrial mean pressures were significantly associated with AA $(P=.01)$ but not with VT/VF $(P=.07)$. Similarly, pulmonary capillary wedge pressures were associated with AA $(P=.02)$ but not with VT/VF $(P=.31)$. Animals in the $\mathrm{PAB}, \mathrm{PV}$, and $\mathrm{PAB} / \mathrm{PV}$ groups had significantly higher right atrial mean pressures and significantly higher capillary wedge pressures than animals in the control groups (Table 1).

\section{Discussion}

Despite the excellent current status of corrective repair of TOF, late SCD occurs in a significant number of patients and has been attributed to lethal ventricular arrhythmias. There has also been some suggestion that AAs might be lethal in these patients as well. In one of the largest cohort studies, Gatzoulis and colleagues ${ }^{9}$ showed an exponentially increased incidence of VT/VF and SCD with time, reporting an $11.9 \%$ incidence of $\mathrm{VT} / \mathrm{VF}$ and an $8.3 \%$ occurrence of SCD by age 35 years. In the most recent multicenter cohort of 252 patients, Khairy and associates ${ }^{7}$ documented the occurrence of clinical VT/VF, SCD, or both in $24.6 \%$ of patients.

Over the past several decades, considerable effort has been made both to identify the substrate for these arrhythmias and to stratify patients according to their risk of VT/VF and SCD. Potential risk factors for the development of ventricular arrhythmias have been identified and include (1) older age at repair and longer duration of postsurgical follow-up, ${ }^{14-16}$ (2) frequent ectopic beats, ${ }^{14,17}$ (3) increased RV systolic pressure, ${ }^{13-15}$ (3) increased RV diastolic pressure, ${ }^{13,14}$ (4) conduction alterations in regions of myocardial fibrosis, ${ }^{18-21}$ (5) moderate or severe PR with resultant RV dilatation, ${ }^{9,15,17,22-24}$ (6) increased duration of ventricular depolarization as recorded by QRS duration, ${ }^{7,9,22}$ and (7) inducible VT/VF during programmed ventricular stimulation. ${ }^{7,26,27}$ Although it is likely that the development of malignant arrhythmias in the patient with TOF postoperatively is multifactorial, there have been no controlled prospective studies designed to isolate these individual risk factors and determine their independent contribution to the development of significant arrhythmias. In this animal study, a distinct model of physiologic states in postoperative TOF was reliably reproduced with RV hypertension, RV dilation, and scarring in the RV outflow tract. These models were used to study the effects of each independent variable 
and their relative contribution to the development of late inducible lethal arrhythmias.

In this animal model there was an increased incidence of ventricular arrhythmias in those animals that underwent the $\mathrm{PV}$ procedure compared with control animals (OR, 30; $P=$ .011). Neither the Scar group nor the PAB group was statistically more likely to have VT/VF (OR, 2.4 and 1.8, respectively) compared with the control group. The animals that were subject to the combined procedure (PAB/PV) had a higher likelihood of VT/VF inducibility compared with the PAB group animals, but this did not reach significance $(\mathrm{OR}, 4 ; P=.24)$. The single hemodynamic variable that predicted VT/VF inducibility was an increased RV enddiastolic pressure.

The importance of RV volume overload and dilation, presumably caused by PR, tricuspid valve regurgitation, or both, has been previously suggested as a significant risk factor in the development of ventricular arrhythmias. Gatzoulis and colleagues ${ }^{9}$ found that QRS duration was related to RV dilation and that a QRS duration in excess of $180 \mathrm{~ms}$ was predictive of ventricular arrhythmias. No patient with a QRS duration of less than $180 \mathrm{~ms}$ had sustained VT/VF or SCD (100\% sensitive, $94.7 \%$ specific). A recent multicenter analysis reported that a QRS duration of greater than $180 \mathrm{~ms}$ likewise predicted inducibility of VT/VF with programmed ventricular stimulation $(P<.0001){ }^{7}$ This study also indicates that $\mathrm{QRS}$ prolongation is a risk factor for $\mathrm{VT} / \mathrm{VF}$ $(P<.01)$.

This study demonstrated that there is a significant incidence of inducible AAs in this study population, even in the absence of a surgical atriotomy. There was a strong relationship between the type of surgical procedure performed and the ability to induce AA because animals having PAB or $\mathrm{PV}$ procedures were much more likely to have inducible AA compared with the control group. Alterations in atrial and ventricular compliance play a significant role in the development of AA in the postoperative patient with TOF.

It is common practice at many centers to entirely relieve $\mathrm{RV}$ outflow stenosis at the subvalvar and valvar levels, with accepted consequences of creating PR and chronic volume overload. ${ }^{28}$ This study suggests that RV dilatation is arrhythmogenic and predisposes subjects to potentially lethal arrhythmias. It is possible that patients with TOF might benefit from less aggressive measures to relieve pulmonary stenosis and to maintain the integrity of the pulmonary valve apparatus after the initial repair. Alternative surgical approaches that would allow better preservation of pulmonary valve leaflets and limit the degree of PR should be considered. Parry and coworkers ${ }^{29}$ reported the success of a limited transannular patching technique in relieving RV outflow tract obstruction with the purpose of preserving pulmonary valvar function as much as possible. Furthermore, early pulmonary valve replacement should be consid- ered for those patients before progressive RV dilation in an attempt to protect them from the potential insult of PR and the risk of SCD. Therrien and associates ${ }^{30}$ found that pulmonary valve replacement in this patient population led to the stabilization of the QRS duration and, with concurrent intraoperative cryoablation, a significant reduction in the incidence of both atrial and ventricular arrhythmias. Recently, interest has developed in newer approaches, such as the placement of percutaneous pulmonary valves ${ }^{31}$ in the cardiac catheterization laboratory, to reestablish pulmonary valve competency while avoiding the surgical risk inherent with reoperation.

\section{Study Limitations}

Although this study creates similar physiology to postoperative TOF, it does not include the anatomic or genetic substrate, such as malalignment of the ventricular septum, ventricular septal defect, or subvalvar pulmonary stenosis. Any additional contribution of the anatomy and genetic alterations to the development of late arrhythmias are not accounted for in this study. A final limitation in the design of the study is the duration of follow-up after neonatal cardiac surgery. Although this study follows the animals to early adulthood, late-onset lethal arrhythmias might progress more insidiously and do not become manifest until late adulthood.

\section{Conclusions}

In this prospective animal study a reproducible pig model was created to study the roles of RV hypertension, severe $\mathrm{PR}$, and scarring in the RV outflow tract and their contribution to the development of atrial and ventricular arrhythmias. In this model, chronic RV volume overload and RV dilation caused by free PR appear to establish the necessary electrophysiologic and hemodynamic conditions to induce and sustain significant atrial and ventricular tachyarrhythmias. Furthermore, residual hemodynamic lesions resulting in an increased RV end-diastolic pressure are associated with an increased incidence of inducible arrhythmias. Lastly, RV hypertension and RV dilation both appear related to the development of AAs. These results suggest that aggressive management strategies aimed at reducing RV volume burden, size, or both are warranted in patients who have undergone surgical correction of TOF with the goal to prevent VT/VF and SCD.

We thank Peggy McCann for her assistance in obtaining the echocardiographic images, David Riffe for assistance in recording numerous ECGs, and David Donnelley for technical support during the invasive hemodynamic evaluations.

\section{References}

1. Perry LW, Neill CA, Ferencz C, et al. Infants with congenital heart disease: the cases. In: Ferencz C, Rubin JD, Loffredo CA, et al, editors. 
Perspectives in pediatric cardiology. epidemiology of congenital heart disease, the Baltimore-Washington Infant Study 1981-1989. Armonk, NY: Futura; 1993. p. 33-62.

2. Hennein HA, Mosca RS, Ureclay G, Crowley DC, Bove EL. Intermediate results after complete repair of tetralogy of Fallot in neonates. J Thorac Cardiovasc Surg. 1995;109:332-42.

3. Murphy JG, Gersh BJ, Mair DD, et al. Long-term outcome in patients undergoing surgical repair of tetralogy of Fallot. $N$ Engl J Med. 1993;329:593-9.

4. Garson A. Ventricular arrhythmias after repair of congenital heart disease: who needs treatment? Cardiol Young. 1991;1:177-81.

5. Gillette PC, Yeoman MA, Mullins CE, McNamara DG. Sudden death after repair of tetralogy of Fallot. Circulation. 1977;56:566-70.

6. Dunnigan A, Pritzker MR, Benditt DG, Benson DW. Life threatening ventricular tachycardias in late survivors of surgically corrected tetralogy of Fallot. Br Heart J. 1984;52:198-206.

7. Khairy P, Landzberg MJ, Gatzoulis MA, et al. Value of programmed ventricular stimulation after tetralogy of Fallot: a multicenter study. Circulation. 2004;109:1994-2000.

8. Gillette PC, Garson A. Sudden cardiac death in the pediatric population. Circulation. 1992;85(suppl I):I64-9.

9. Gatzoulis MA, Balaji S, Webber SA, et al. Risk factors for arrhythmia and sudden cardiac death late after repair of tetralogy of Fallot: a multicentre study. Lancet. 2000;356:975-81.

10. Nollert G, Fischlein T, Bouterwk S, Bohmer C, Klinner W, Reichart B. Long-term survival in patients with repair of tetralogy of Fallot: 36-year follow-up of 490 survivors of the first year after surgical repair. J Am Coll Cardiol. 1997;30:1374-83.

11. Bricker JT. Sudden death and tetralogy of Fallot: risks, markers, and causes. Circulation. 1995;92:158-9.

12. Jonsson H, Ivert T, Brodin LA. Late sudden deaths after repair of tetralogy of Fallot. Scand J Thorac Cardiovasc Surg. 1995;29:131-9.

13. Garson A, Nihill MR, McNamara DG, Cooley DA. Status of the adult and adolescent after repair of tetralogy of Fallot. Circulation. 1979; 59:1232-40.

14. Chandar JS, Wolff GS, Garson A, et al. Ventricular arrhythmias in postoperative tetralogy of Fallot. Am J Cardiol. 1990;65:655-61.

15. Marie PY, Marcon F, Brunotte F, et al. Right ventricular overload and induced sustained ventricular tachycardia in operatively "repaired" tetralogy of Fallot. Am J Cardiol. 1992;69:785-9.

16. Kavey RW, Blackman MS, Sondheimer HM. Incidence and severity of chronic ventricular dysrhythmias after repair of tetralogy of Fallot. Am Heart J. 1982;103:342-50.
17. Harrison DA, Harris L, Siu SC, et al. Sustained ventricular tachycardia in adult patients late after repair of tetralogy of Fallot. J Am Coll Cardiol. 1997;30:1368-73.

18. Deanfiled JE, Ho SY, Anderson RH, McKenna WJ, Allwork SP, Hallidie-Smith KA. Late sudden death after repair of tetralogy of Fallot: a clinicopathologic study. Circulation. 1983;67:626-31.

19. Horowitz LN, Vetter VL, Harken AH, Josephson ME. Electrophysiologic characteristics of sustained ventricular tachycardia occurring after repair of tetralogy of Fallot. Am J Cardiol. 1980;46:446-51.

20. Downar E, Harris L, Kimber S, et al. Ventricular tachycardia after surgical repair of tetralogy of Fallot: results of intraoperative mapping studies. J Am Coll Cardiol. 1992;20:648-55.

21. Gonska BD, Cao K, Raab J, Kreuzer H. Radiofrequency catheter ablation of right ventricular tachycardia late after repair of congenital heart defects. Circulation. 1996;94:1902-8.

22. Gatzoulis MA, Till JA, Somerville J, Redington AN. Mechanoelectrical interaction in tetralogy of Fallot. Circulation. 1995;92:231-7.

23. Zahka KG, Horneffer PJ, Rowe SA, et al. Long-term valvular function after total repair of tetralogy of Fallot. Circulation. 1988;78(suppl III):III14-9.

24. Hansen DE, Craig CS, Hondeghem LM. Stretch-induced arrhythmias in the isolated canine ventricle. Circulation. 1990;81:1094-104.

25. Swindle MM, Smith AC, Hepburn BJS. Swine as models in experimental surgery. J Invest Surg. 1988;1:65-79.

26. Garson A, Porter CO, Gillette PC, McNamara DG. Induction of ventricular tachycardia during electrophysiologic study after repair of tetralogy of Fallot. J Am Coll Cardiol. 1983;1:1493-502.

27. Alexander ME, Walsh EP, Saul JP, Epstein MR, Triedman JK. Value of programmed ventricular stimulation in patients with congenital heart disease. J Cardiovasc Electrophysiol. 1999;10:1033-44.

28. Castaneda AR, Jonas RA, Mayer JE, Hanley FL. Tetralogy of Fallot. In: Cardiac Surgery of the Neonate and Infant. Philadelphia, Pa: W.B. Saunders Company; 1984. p. 215-34.

29. Parry AJ, McElhinney DB, Kung GC, Reddy M, Brook MM, Hanley FL. Elective primary repair of acyanotic tetralogy of Fallot in early infancy: overall outcome and impact on the pulmonary valve. $J \mathrm{Am}$ Coll Cardiol. 2000;36:2279-83.

30. Therrien J, Siu SC, Harris L, et al. Impact of Pulmonary valve replacement on arrhythmia propensity late after repair of tetralogy of Fallot. Circulation. 2001;103:2489-94.

31. Boudjemline Y, Agnoletti G, Bonnet D, Sidi D, Bonhoeffer P. Percutaneous pulmonary valve replacement in a large right ventricular outflow tract: an experimental study. J Am Coll Cardiol. 2004;43: 1082-7. 